\title{
Links Between Strokes and Hereditary Hemorrhagic Telangiectasia: A Population-Based Study
}

\author{
Farah N. Chowdhury, G. Sanjaya Chandrarathne, Kristopher D. Masilamani, \\ Jennifer T. N. LaBranche, Shaun Malo, Lawrence W. Svenson, Thomas Jeerakathil, \\ Dilini P. Vethanayagam
}

\begin{abstract}
Background: Hereditary hemorrhagic telangiectasia (HHT) is a disease of abnormal vasculature where patients are predisposed to strokes of multiple etiologies. We assessed yearly stroke incidence among Albertans with HHT and compared with the general population. Given the tendency for stroke in HHT patients, we expected HHT patients to have higher stroke incidence, in particular at younger ages. Methods: Population-based administrative health data on inpatient and ambulatory admissions were extracted over a 16-year period using International Classification of Diseases (ICD)-9 and ICD-10, Canada codes. We analyzed overall occurrence of strokes in Alberta by age, gender, stroke subtype, and diagnosis of HHT. Results: The age-standardized incidence rate of stroke in HHT was 450 per 100,000 compared with 260 per 100,000 in the general population with a rate ratio of 1.73 (95\% confidence interval (CI) [1.046-2.842]). This study found a higher HHT prevalence in Alberta (1 in 3800) compared to the world average of 1 in 5000. Women were also more likely to be diagnosed with HHT, with a 3.25:1 female gender preponderance in the yearly incidence. Interpretation: This study not only shows that HHT patients are at higher risk of having a stroke but also quantifies that risk using an age-adjusted metric in Alberta. This province has a higher than expected disease burden of HHT, with the majority of cases affecting women. Our study found that acute ischemic strokes and transient ischemic attacks are far more common than hemorrhage in HHT. As HHT is a rare, multi-system, chronic disease, these patients should be referred to an HHT Centre of Excellence.
\end{abstract}

RÉSUMÉ: Les liens existant entre les AVC et la maladie de Rendu-Osler-Weber: une étude basée sur la population albertaine. Contexte: La maladie de Rendu-Osler-Weber (MROW) est une maladie caractérisée par une anomalie du système vasculaire. Les patients qui en sont atteints deviennent prédisposés à être victimes d'AVC présentant de multiples étiologies. À cet égard, nous avons cherché à évaluer l'incidence annuelle d'AVC au sein de patients albertains atteints de la MROW comparativement à l'incidence annuelle d'AVC au sein de la population de cette province. Étant donné la prédisposition évoquée ci-haut, nous nous attendions à ce que l'incidence d'AVC chez des patients atteints de cette maladie soit plus élevée, particulièrement à un plus jeune âge. Méthodes: Des données administrative liées à la santé et basées sur une population déterminée ont été extraites au moyen des codes ICD-9 et ICD-10-CA. Elles concernaient des patients hospitalisés et ambulatoires au cours d'une période de 16 ans. Nous avons ensuite analysé l'incidence d'ensemble des AVC en Alberta en fonction de l'âge, du sexe, des sous-types d'AVC et de l'établissement préalable d'un diagnostic de MROW. Résultats: Le taux d'incidence normalisé selon l'âge des AVC chez les patients atteints de la MROW était de 450 pour 100000 comparativement à 260 pour 100000 au sein de la population générale. Le ratio des taux, lui, était de 1,73 (IC $95 \%$ [1,046;2,842]). Notre étude a aussi constaté une prévalence plus élevée de la MROW en Alberta (1 sur 3 800) comparativement à la moyenne mondiale, qui est de 1 sur 5 000. Fait à souligner, les femmes étaient plus susceptibles de recevoir un diagnostic de MROW (prépondérance féminine de 3,25:1 sur une base annuelle). Interprétation: Cette étude a montré que les patients atteints de la MROW étaient non seulement plus à risque d'être victimes d'un AVC mais a aussi quantifié ce risque au moyen d'un outil de mesure ajusté pour l'Alberta en fonction de l'âge. Cette province donne à voir une incidence plus élevée de MROW que ce à quoi on pouvait s'attendre, la majorité des patients étant de sexe féminin. Notre étude a en outre montré que les AVC ischémiques aigus et les accidents ischémiques transitoires (AIT) sont nettement plus courants que les hémorragies lorsqu'on est atteint de la MROW. Considérant qu'il s'agit d'une maladie chronique rare de nature multisystémique, ceux et celles qui en sont atteints devraient être dirigés vers un centre d'excellence spécialisé dans son traitement.

Keywords: Embolic strokes, Stroke, Transient ischemic attack, Hereditary hemorrhagic telangiectasia, Arteriovenous malformation doi:10.1017/cjn.2018.360 Can J Neurol Sci. 2019; 46: 44-50

From the Department of Medicine, Division of Pulmonary, University of Alberta, Edmonton, Alberta, Canada (FNC, GSC, KDM, JTNL, DPV); Department of Physiology and Neurobiology, University of Connecticut, Storrs, Connecticut, USA (KDM); Analytics and Performance Reporting Branch, Alberta Health, Edmonton, Alberta, Canada (SM, LWS); Division of Neurology, University of Alberta, Edmonton, Alberta, Canada (TJ); School of Public Health, University of Alberta, Edmonton, Alberta, Canada (LWS); Department of

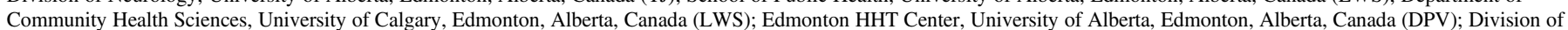
Preventive Medicine, University of Alberta, Edmonton, Alberta, Canada (LWS)

Received December 19, 2017. Final Revisions Submitted August 3, 2018. Date of Acceptance September 10, 2018.

Correspondence to: Dilini P. Vethanayagam, 3-105 Clinical Sciences Building, University of Alberta, 11350-83 Avenue, Edmonton, Alberta, Canada T6G 2G3.

Email: dilini@ualberta.ca 


\section{INTRODUCTION}

Hereditary hemorrhagic telangiectasia (HHT) is an autosomal dominant genetic disorder with an estimated prevalence of 1 in 5000 people in North America, ${ }^{1}$ the majority of whom have not been screened at an HHT screening and treatment center. This disorder is associated with abnormal vascular development and organ-specific over-activity of vascular endothelial growth factor, the mechanisms of which have not been fully elucidated. The most common clinical manifestations include spontaneous recurrent epistaxis, mucocutaneous telangiectasia, and most importantly, visceral arteriovenous malformations (AVMs) particularly prevalent in the brain, liver, and pulmonary circulation. ${ }^{2}$ It is estimated that $35 \%-40 \%$ of individuals with HHT have pulmonary AVMs (PAVMs) and 10\% have cerebral AVMs (CAVMs). ${ }^{3,4}$

There is limited data on the risk for both hemorrhagic stroke and ischemic stroke in HHT in comparison to the general population. A study in a large HHT clinic, comprising approximately $10 \%$ of all HHT cases in the United Kingdom, found a 16-fold increase in hemorrhagic stroke risk and a seven-fold increase in ischemic stroke risk compared to the general population. ${ }^{3}$ Presence of AVMs increases the likelihood of a stroke occurrence in an individual; studies have shown that there is a higher correlation of PAVMs with ischemic stroke and CAVMs with hemorrhagic events. $^{5-8}$

Hereditary hemorrhagic telangiectasia is unusual in that the unique vascular pathophysiology makes it one of the few known genetic conditions that can lead to hemorrhagic, thrombotic, and embolic strokes. ${ }^{9-11}$ Precise estimates of risk are impeded by the rarity of the condition and the lack of population-based data on risk assessment. Using a province-wide, population-based, administrative health data set, we examined the stroke risk in patients with HHT compared to the general population.

\section{METHODS}

We conducted a retrospective population-based study using information from the Alberta Health $(\mathrm{AH})$ databases. As Alberta maintains a publicly funded, universally available, healthcare system, residents of the province must register with the Alberta Health Care Insurance Plan (AHCIP). Each registrant with the AHCIP is issued a person health number that acts as a unique lifetime identifier, and is captured in all interactions with the healthcare system. Alberta has a population-based, provincewide, discharge abstracts database which includes all inpatient hospitalizations, ambulatory hospital visits, and emergency room visits for patients of all ages. The province also maintains a comprehensive physician claims database which captures physician visits for inpatient and outpatient settings and associated International Classification Disease (ICD)-10 diagnostic codes. Alberta Ministry of Health staff extracted the data and provided statistical analysis.

Inclusion criteria for this project were all strokes (both in pediatric and adult patients) in Alberta as captured through the Alberta Health databases who presented to an emergency department and/or were hospitalized in the province of Alberta between 1997 and 2012. We excluded any individuals who did not meet either of the above criteria.

To have an HHT diagnosis for the purposes of this study, all individuals captured required two different physician clinic visits within one year with a diagnosis of HHT (ICD-09: 448.0) or two outpatient hospital visits or emergency room visits, each with a diagnosis of HHT (ICD-09: 448.0), or one inpatient visit with a diagnosis of HHT (ICD-09: 448.0). The more stringent definition of requiring two physician visits to confirm the HHT diagnosis (although not requiring the physicians to be HHT specialists) ensures the dataset errs on the side of high specificity for HHT.

The AH databases were reviewed from 1997 to 2012 for four major forms of stroke-related codes presenting to the emergency department and/or requiring hospital admissions: acute ischemic stroke (AIS), intracerebral hemorrhage (ICH), subarachnoid hemorrhage (SAH), and transient ischemic attack (TIA). The HHT diagnoses were correlated through this same registry. We retrieved patient age and gender demographics with all stroke admissions. Patients were sub-divided by gender into five age groups: Birth, 0-17 years, 18-30 years, 31-60 years, and over 60 years of age.

The incidence of stroke was captured using diagnostic codes: ischemic stroke (ICD-10: I63.9), non-traumatic subarachnoid hemorrhage (ICD-10: I60.0), non-traumatic (ICD: I61.9), and TIAs (ICD-10: G45.9). ${ }^{27}$ All strokes among the HHT patient group were captured in a similar manner and incidence rates per 100,000 person-years calculated for each age group.

\section{Statistical Analysis}

Descriptive statistics were used to calculate the stroke incidence rate in both HHT and non-HHT groups. The direct method was used for age-standardization, and standardized rate ratio (SRR) was used to compare the stroke incidence rates between HHT and non-HHT population groups. Excel for Mac (version 15.26 [2016], Microsoft Corporation, Redmond, WA, USA) was used to calculate the SRR. Incidence rate ratios of each stroke subtype in both the HHT and general population were calculated with confidence intervals using the same software.

\section{RESULTS}

From the years 1997 to 2012, we found a significantly higher incidence of strokes in patients with HHT compared to the baseline population. The stroke incidence rate in HHT was about 450 per 100,000 people as compared to 260 per 100,000 people in the general population (Figure 1). The proportions of each stroke subtype were the same in both groups. The majority of strokes

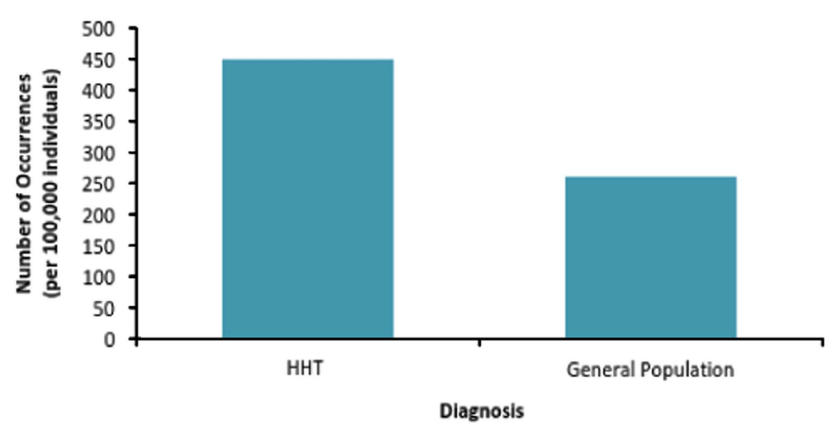

Figure 1: Age-standardized stroke incidence rate in HHT and nonHHT population in Alberta. Stroke incidence rate in Albertans with HHT-450 per 100,000 person-years (95\% CI [226-673]) compared with 260 per 100,000 person-years (95\% CI [259-262]) in the general population. 
Table 1: Stroke subtypes in HHT and non-HHT groups

\begin{tabular}{|c|c|c|c|c|}
\hline & \multirow{2}{*}{$\begin{array}{c}\text { Incidence } \\
\text { AIS }\end{array}$} & \multirow{2}{*}{$\begin{array}{c}\text { Incidence rate per 100,000 person-years } \\
\text { AIS }\end{array}$} & \multicolumn{2}{|c|}{ Normal approximation $95 \%$ confidence interval $(\mathrm{CI})$} \\
\hline & & & & \\
\hline HHT incidence & 40 & HHT & Lower CI & Upper CI \\
\hline HHT person-year & 10,648 & 375.66 & 259.24 & 492.07 \\
\hline Non-HHT incidence & 65,534 & Non-HHT & Lower CI & Upper CI \\
\hline \multirow[t]{2}{*}{ Non-HHT person-year } & $53,738,064$ & 121.95 & 121.02 & 122.88 \\
\hline & $\mathrm{ICH}$ & $\mathrm{ICH}$ & & \\
\hline HHT incidence & 3 & HHT & Lower CI & Upper CI \\
\hline HHT person-year & 10,648 & 28.17 & $5.81 *$ & $82.34 *$ \\
\hline Non-HHT incidence & 7597 & Non-HHT & Lower CI & Upper CI \\
\hline \multirow[t]{2}{*}{ Non-HHT person-year } & $53,738,064$ & 14.14 & 13.82 & 14.15 \\
\hline & SAH & SAH & & \\
\hline HHT incidence & 3 & HHT & Lower CI & Upper CI \\
\hline HHT person-year & 10,648 & 28.17 & $5.81 *$ & $82.34 *$ \\
\hline Non-HHT incidence & 5978 & Non-HHT & Lower CI & Upper CI \\
\hline \multirow[t]{2}{*}{ Non-HHT person-year } & $53,738,064$ & 11.12 & 10.84 & 11.41 \\
\hline & TIA & TIA & & \\
\hline HHT incidence & 32 & HHT & Lower CI & Upper CI \\
\hline HHT person-year & 10648 & 300.52 & 196.40 & 404.65 \\
\hline Non-HHT incidence & 52088 & Non-HHT & Lower CI & Upper CI \\
\hline \multirow[t]{2}{*}{ Non-HHT person-year } & $53,738,064$ & 96.93 & 96.10 & 97.76 \\
\hline & All strokes & All strokes & & \\
\hline HHT incidence & 78 & HHT & Lower CI & Upper CI \\
\hline HHT person-year & 10648 & 732.53 & 569.96 & 895.10 \\
\hline Non-HHT incidence & 131197 & Non-HHT & Lower CI & Upper CI \\
\hline Non-HHT person-year & $53,738,064$ & 244.14 & 242.82 & 245.46 \\
\hline
\end{tabular}

AIS $=$ acute ischemic stroke, $\mathrm{ICH}=$ intracranial hemorrhage, $\mathrm{SAH}=$ subarachnoid hemorrhage, TIA $=$ transient ischemic attack.

The cumulative incidence of different types of stroke in patients with and without HHT over 16 years. The adjacent column shows the ratio of this incidence by 100,000 person-years for comparison between the HHT and non-HHT groups with CI.

*Exact CI used for small case counts (under 20).

were AIS, which accounted for about $52 \%$ of all strokes in the diagnosed HHT group and about $50 \%$ of all strokes in the nonHHT group (Table 1). Transient ischemic attacks were the second most frequent type in both populations (41\% versus $40 \%$ ). Notably, rates of each stroke subtype were higher in the HHT population than in the general population when ratios of incidence to person-years are compared.

In both groups $<3 \%$ of strokes occurred under 30 years of age. In the diagnosed HHT group, $21 \%$ of strokes occurred in the middle-age group (31-60 years), and $76 \%$ in the elderly group ( $>60$ years). These percentage breakdowns for stroke by age groups are nearly the same for the non-HHT population as well (0-17 years and 18-30 years: 2\%, 31-60 years: $23 \%$, and over 60 years: $75 \%$ ). Figure 2 illustrates the rising incidence of HHT over 16 years in the Alberta population.

Three key trends emerged from our data on increased burden of disease in Alberta: a definite female predilection for HHT diagnosis, a high incidence of stroke in females with HHT compared to men with HHT, and a higher overall prevalence for HHT in Alberta compared to the worldwide prevalence.
Interestingly, the female to male ratio of HHT incidence averaged over 16 years is 3.25:1 (95\% CI [3.24-3.25]). Moreover, women with HHT were also more likely than men to have strokes at a 1.88:1 female to male ratio. There is an overall HHT prevalence of 1 in 3800 in Alberta, which is higher than the estimated 1:5000 worldwide prevalence. These specific gender trends are broken down by stroke subtype by four-year clusters in Table 2.

\section{DISCUSSION}

Stroke occurrence in Alberta is on the rise, likely due to a growing population (from 2.8 million in 1997 to 3.9 million in 2012). ${ }^{12}$ There is a single HHT center for the province. Given Alberta's widely dispersed population, screening individuals for HHT even after a stroke can be difficult. Our study contributed a better understanding of both age and sex-related trends regarding HHT and stroke in Alberta, namely that the age-standardized rate of stroke was significantly higher for persons with HHT compared with the baseline population (Figure 1) and that, despite a wide 
(a)
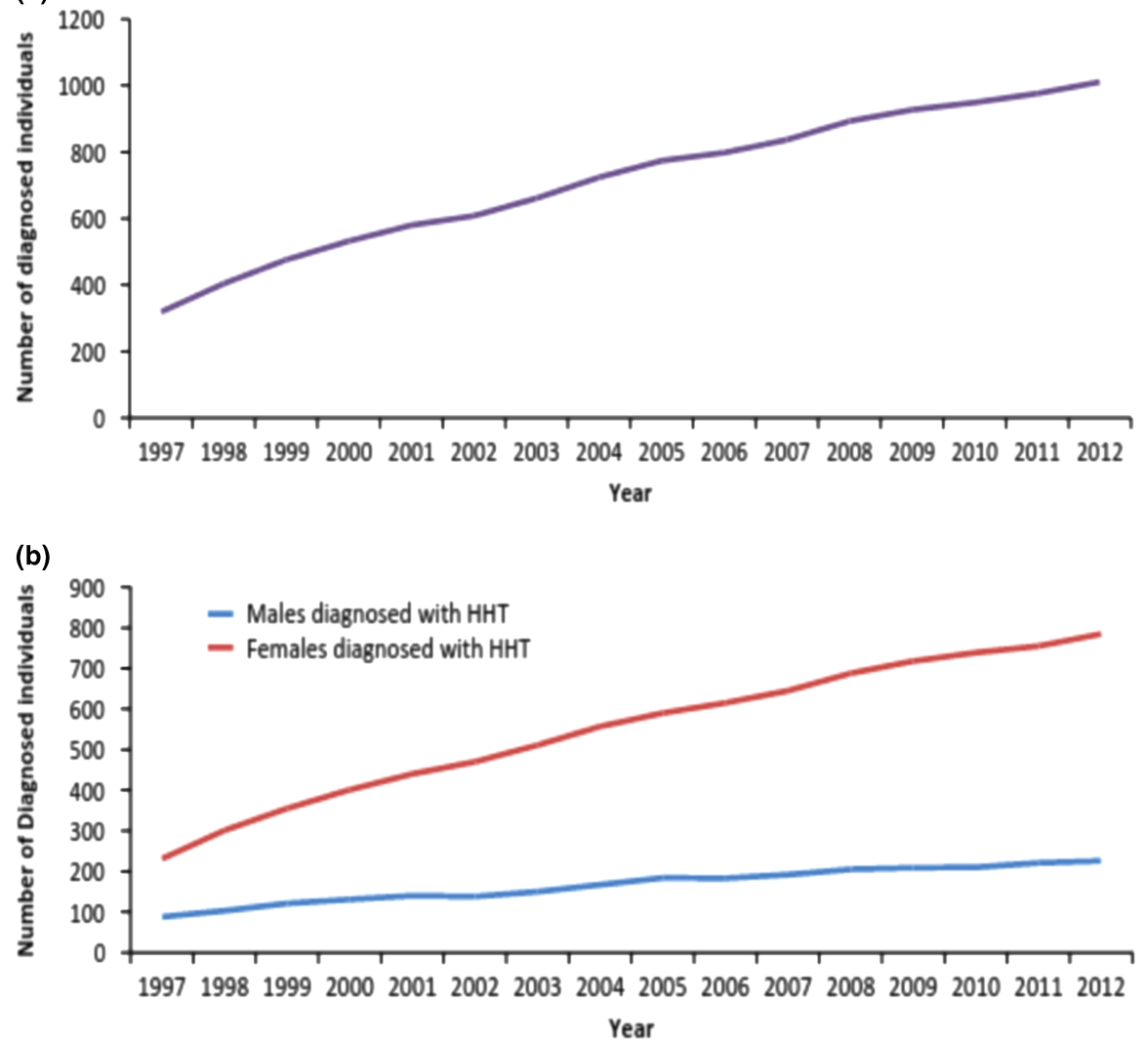

Figure 2: Trends in the total number of patients diagnosed with HHT per year in Alberta between 1997 and 2012. a) Total number of Albertans diagnosed with HHT per year. The overall HHT prevalence in Alberta is about 1 in 3800, compared with the North American estimate of 1 in 5000. b) Albertans diagnosed with HHT classified by gender. Though the total number of individuals diagnosed with HHT is steadily increasing, a closer analysis by gender reveals the male incidence is not growing at the same rate as female incidence per year.

confidence interval and small population size, there is a higher rate of stroke in HHT.

Regardless of age, an individual diagnosed with HHT is 1.73 times more likely to suffer morbidity and mortality from a stroke than if he or she were not diagnosed with HHT. As HHT predisposes patients to strokes via multiple mechanisms, we attribute this trend to the likely higher probability of an underlying genetic component in strokes occurring before age 60. Screening for HHT through an HHT center should be considered in this group in high risk individuals using historical information (epistaxis, telangiectasia, absence of traditional stroke risk factors). This approach may prompt clinicians to identify patients at risk of having HHT before patients present with sentinel diseases such as stroke at a young age. HHT Centers of Excellence are multidisciplinary care centers, specifically accredited by the Cure HHT Foundation of North America.

Another objective of this study was to understand if any particular age groups were more likely to have strokes with concurrent HHT diagnosis. The database yielded limited data on pediatric strokes as only one documented stroke occurred in the diagnosed HHT group. These findings were expected since pediatric strokes are also rare in the general population, thus the data were not adequately powered to elucidate correlations between HHT and strokes in pediatric patients.
The majority of strokes in both HHT and non-HHT populations occur in those over 60 years of age; a considerable portion of these cases were susceptible to cardiovascular risk factors. The lower risk middle-age strata is an important population because strokes occurring in patients between 31 and 60 years of age are more likely to have atypical causes for their stroke including genetic conditions such as HHT. ${ }^{13,23}$

We also found that stroke occurrence is steadily increasing throughout the 16-year span of this study in the non-HHT group, which concurs with findings from other studies that show a growing proportion of strokes occurs in middle-aged patients. ${ }^{14}$ Individuals with strokes occurring in this age range, in the absence of a substantial risk factor burden, may benefit from an assessment for HHT. It is suspected that out of the general population, some individuals who have experienced a stroke within this age group may have HHT, but may have never been screened for the condition.

Our study found HHT to be more common in Alberta than previously reported, at a prevalence of 1 in 3800 . There is a wide range for HHT prevalence worldwide. Populations with Dutch genetic backgrounds seemingly have a higher prevalence than others. One such population comes from the Dutch Antilles, where the highest prevalence of HHT exists at 1 in $1331 .^{15}$ The Alberta prevalence falls within the available published data. There 
Table 2: Total patient numbers for all stroke types for HHT and non-HHT groups per 4-year cluster

\begin{tabular}{|c|c|c|c|c|c|}
\hline \multirow[b]{2}{*}{ Gender } & \multirow[b]{2}{*}{ Stroke subtype } & \multicolumn{4}{|c|}{ Four-year cluster } \\
\hline & & 1997-2000 & 2001-2004 & 2005-2008 & 2009-2012 \\
\hline \multirow[t]{2}{*}{ Female } & AIS in HHT & 5 & 5 & 3 & 13 \\
\hline & AIS in non-HHT & 7792 & 8012 & 7913 & 8389 \\
\hline \multirow[t]{2}{*}{ Male } & AIS in HHT & 2 & 5 & 5 & 2 \\
\hline & AIS in non-HHT & 7974 & 8226 & 8296 & 8932 \\
\hline \multirow[t]{2}{*}{ Female } & ICH in HHT & 0 & 0 & 0 & 1 \\
\hline & $\mathrm{ICH}$ in non-HHT & 876 & 924 & 882 & 924 \\
\hline \multirow[t]{2}{*}{ Male } & $\mathrm{ICH}$ in $\mathrm{HHT}$ & 1 & 0 & 1 & 0 \\
\hline & ICH in non-HHT & 979 & 950 & 1013 & 1049 \\
\hline \multirow[t]{2}{*}{ Female } & SAH in HHT & 0 & 1 & 0 & 1 \\
\hline & SAH in non-HHT & 915 & 960 & 848 & 808 \\
\hline \multirow[t]{2}{*}{ Male } & SAH in HHT & 1 & 0 & 0 & 0 \\
\hline & $\mathrm{SAH}$ in non-HHT & 600 & 665 & 612 & 570 \\
\hline \multirow[t]{2}{*}{ Female } & TIA in HHT & 3 & 6 & 6 & 7 \\
\hline & TIA in non-HHT & 6386 & 7006 & 6667 & 6789 \\
\hline \multirow[t]{2}{*}{ Male } & TIA in HHT & 2 & 3 & 2 & 3 \\
\hline & TIA in non-HHT & 5826 & 6412 & 6296 & 6706 \\
\hline
\end{tabular}

AIS = acute ischemic stroke, $\mathrm{ICH}=$ intracranial hemorrhage, $\mathrm{SAH}=$ subarachnoid hemorrhage, TIA = transient ischemic attack.

Incidences of each stroke subtype for males and females with and without HHT in Alberta over four-year clusters spanning the period of this study.

are many Dutch settlements in Alberta ${ }^{16}$ and many rural communities with large family units affected by HHT.

Another significant finding of this study includes the difference between males and females who have been diagnosed with HHT. Interestingly, over the 16 years of our study, females were on average 3.25 times more likely to be diagnosed with HHT. Other studies have shown similar trends, however, not quite as pronounced difference as our data suggests. An HHT populationbased study by Donaldson et al found the female to male ratio for HHT diagnosis to be $1.7: 1 .{ }^{17}$ Our results do not accurately portray the genetic nature of HHT: it is not sex-linked. This discrepancy, which is unexpected given the autosomal inheritance of HHT, points to two possible reasons that the data show a female gender preponderance and males are being under-diagnosed or females may experience higher penetrance and thus are more symptomatic at the population level.

There are a number of explanations for the discrepancy between our results and the literature with regard to HHT prevalence in females. Hereditary hemorrhagic telangiectasia underdiagnosis in males is a likely contributor as clinical vigilance for rare diseases like HHT is often inadequate. Signs and symptoms of HHT may escape a clinician's suspicion because they are often obscure or non-specific, such as chronic epistaxis, occult gastrointestinal bleeding, telangiectasias in the oral or nasal mucosa, chronic fatigue, or headaches. Moreover, probably men are less likely to bring such complaints to their primary care provider's attention as compared with women. These views are supported by the Statistics Canada report "Women and Health" that showed females are more likely than males to have a family physician and are also more likely to seek medical attention. ${ }^{18}$

Outside of these behavioral considerations, existing literature suggests women are also more likely to have more severe symptoms in HHT. Females have a greater physiological potential to present with bleeding manifestations given that they are prone to all of the bleeding opportunities of males (such as epistaxis and gastrointestinal hemorrhage) as well as uterine bleeding. The combined likelihood of hemorrhage could also increase the chances of presenting to medical care because of iron deficiency anemia. Shovlin et al identified patients to be particularly susceptible to cerebral ischemia if they have iron deficiency anemia in HHT. ${ }^{19}$ Accordingly, we see that women in Alberta with HHT are 1.88 times more likely that males to have a stroke or TIA. Another factor that makes women more vulnerable to severe complications of HHT is pregnancy. This is especially true when obstetricians and patients have no prior knowledge of an HHT diagnosis, so complications such as PAVM hemorrhage, stroke, and maternal death proceed in about $1 \%$ of pregnancies in mothers with HHT without the added benefit of clinical vigilance. ${ }^{20}$

Beyond age and gender trends, our study quantified rates of stroke subtypes in HHT in Alberta. There are multiple etiologies conferring strokes and transient ischemia upon HHT patients. Acute ischemic stroke was the most frequent subtype of stroke in the HHT population at 40 events out of 78. Transient ischemic attacks were also quite frequent at 32 events out of 78. Patients with HHT are at particularly high risk for these two stroke subtypes because of key hallmarks of HHT that predispose to cerebral ischemia, such as PAVMs, CAVMs and brain abscess, or thrombus formation. Abnormal vascular maturation in the pulmonary circulation can result in a right-to-left shunt (RLS) from PAVMs. Velthius et al found a significant correlation between higher-grade pulmonary RLS and cerebral complications $(21 \%) .{ }^{21}$ Ischemic strokes and TIAs may be seen in individuals with HHT who have PAVMs with larger feeding arteries and not treated with standardized thoracic embolization protocols. Patients who have both 
CAVMs and PAVMs are also at particularly high risk of cerebral abscess that can lead to ischemia since PAVMS lack the capillary beds that often filter out typical lung pathogens. ${ }^{9}$ Mathis et al postulate that emboli from PAVMs produce cerebral infarcts where hypoxia and polycythemia produce the necessary conditions for abscess development. ${ }^{22}$ Patients with CAVMs in the context of HHT are at particular risk of thrombus formation. This is a reactive process within the CAVM because of hemodynamic changes that may diminish perfusion, exacerbate venous congestion, and lead to stasis and thrombosis. ${ }^{23,24}$

In contrast to ischemic strokes, cerebral hemorrhage was rare in the HHT group, with three cases each of intracranial and SAH, respectively. This finding contrasts the non-population based, but clinically rigorous study by Easey et al found a higher risk of hemorrhagic stroke in a retrospective cohort of HHT probands and their families. ${ }^{3}$ Hereditary hemorrhagic telangiectasia patients are theoretically at risk of hemorrhage as well, since rupture of abnormal intracerebral vasculature from CAVMs can lead to hemorrhagic strokes. ${ }^{25,26}$ These discrepancies in findings might be partially explained by the ease of diagnosis of hemorrhagic stroke and the condition being less subjective to recall bias than ischemic stroke in the retrospective design. ${ }^{3}$ Alternatively, our population-based design would be unlikely to miss cases of ICH that would be very likely to present to emergency departments. However, we may be subjected to misclassification bias and may fail to identify some persons with ICH having probable or possible HHT.

The strength of this study is the population-based design allowing examination of databases of an entire province. Limitations of this study include relatively small number of HHT cases because of the strict method of defining HHT diagnosis. This more stringent definition means we are erring on the side of specificity, biasing toward missing HHT rather than overestimating the rate of HHT. Consequently, our estimates of HHT cases and strokes within the HHT population are conservative. Using a longer period for this study or including more provinces may increase the sample size to show differences between stroke subtypes or age groups more clearly. As HHT is a rare disease, the administrative health data set represents an efficient and costeffective way to estimate prevalence and impact.

Another shortcoming of this study is the lack of data on AVMs in those patients who experienced strokes. Correlating PAVM and CAVM data with stroke subtypes would offer an added robustness to the study methodology. As similar ratios of different stroke subtypes were seen in the non-HHT population as well, it is difficult to postulate without this information, how much these mechanisms of stroke in HHT factored into the risk of stroke for HHT patients compared with the baseline population. Another limitation of our design is the lack of integration with other provincial data. For example, even if one or two patients with ICH present to neighboring provinces, this skews our data as there were only three ICH events over 16 years in the HHT population.

In conclusion, stroke incidence rate is higher among individuals with HHT. As the age-adjusted stroke rate is higher in the HHT group, there may be an underlying genetic contribution. As such, affected individuals should be screened by history for additional stroke risk factors. This is especially true for male patients, as there seems to be an under-diagnosis of HHT in males in Alberta or under-presentation of males to HHT centers or stroke centers. Given that women have unique risk factors for stroke (oral contraceptive use, pregnancy, migraine with aura, and hormone replacement therapy), special consideration should be given to women with HHT who possess additional risk factors. As HHT is a rare, multi-system, chronic disease, these patients should be referred to an HHT Center of Excellence.

\section{ACKNOWLEDGEMENTS}

The authors thank Ms. Iris de Guzman for her assistance in the preparation of this manuscript.

\section{FinANCIAL SuPPORT}

None.

\section{DISCLOSURES}

Farah N. Chowdhury, G. Sanjaya Chandrarathne, Kristopher D. Masilamani, Jennifer T.N. LaBranche, Shaun Malo, Lawrence W. Svenson, and Dilini P. Vethanayagam have nothing to disclose.

Thomas Jeerakathil reports grants and personal fees from CIHR, HSFC, AIHS, CSN, University of Alberta Hospital Foundation, and Bayer, outside the submitted work.

\section{REFERENCES}

1. Govani FS, Shovlin CL. Hereditary hemorrhagic telangiectasia: a clinical and scientific review. Eur J Hum Genet. 2009;17:860-71.

2. Dupuis-Girod S, Bailly S, Plauchu H. Hereditary hemorrhagic telangiectasia: from molecular biology to patient care. J Thromb Haemost. 2010;8:1447-56.

3. Easey AJ, Wallace GMF, Hughes JMB, Jackson JE, Taylor WJ, Shovlin CL. Should asymptomatic patients with hereditary hemorrhagic telangiectasia (HHT) be screened for cerebral vascular malformations? Data from 22061 years of HHT patient life. J Neurol Neurosurg Psychiatry. 2003;74:743-8.

4. Faughnan ME, Palda VA, Garcia-Tsao G, et al. International guidelines for the diagnosis and management of hereditary haemorrhagic telangiectasia. J Med Genet. 2011;48:73-87.

5. Blauwblomme T, Bourgeois M, Meyer $P$, et al. Long-term outcome of 106 consecutive pediatric ruptured brain arteriovenous malformation after combined treatment. Stroke. 2014;45:1664-71.

6. National Institute of Health. National Institute of Neurological Disorders and Stroke. Arteriovenous malformations and other vascular lesions of the central nervous system fact sheet. Available at: http://www.ninds.nih.gov/disorders/avms/detail_avms.htm. Accessed December 8, 2016.

7. Meek ME, Meek JC, Beheshti MV. Management of pulmonary arteriovenous malformations. Semin Intervent Radiol. 2011;28:24-31.

8. Woodall NM, McGettigan M, Figueroa R, Gossage JR, Alleyne CH Jr. Cerebral vascular formations in hereditary hemorrhagic telangiectasia. J Neurosurg. 2014;120:87-92.

9. Akers AL, Ball KL, Clancy M, et al. Brain vascular malformation consortium: overview, progress and future directions. J Rare Disord. 2013;1:5-27.

10. Torring PM, Larsen MJ, Kjeldsen AD, Ousager LB, Tan Q, Pernille M. Long non-coding RNA expression profiles in hereditary hemorrhagic telangiectasia. PLoS One. 2014;9(3):e90272.

11. Caplan LR, Bogousslavsky J. Hereditary hemorrhagic telangiectasia. In: Uncommon causes of stroke, 2nd ed. Cambridge: Cambridge University Press; 2008, pp. 109-15.

12. Jeerakathil T, Burridge D, Thompson G, Fang S, Hill MD. The Alberta provincial stroke strategy: a legacy of stroke care for Alberta. Edmonton: Alberta Provincial Stroke Strategy; 2012. Available at: https://open.alberta.ca/publications/9780986798917. Accessed December 15, 2017.

13. Kjeldsen AD, Oxhøj H, Andersen PE, Green A, Vase P. Prevalence of pulmonary arteriovenous malformations (PAVMs) and occurrence of neurological symptoms in patients with hereditary haemorrhagic telangiectasia (HHT). J Intern Med. 2000;248:255-62. 
14. Kissela B, Khoury J, Alwell K, et al. Age at stroke: temporal trends in stroke incidence in a large, biracial population. Neurology. 2012;79(17):1781-7.

15. Westermann C, Rosina A, de Vries V, Coteau P. The prevalence and manifestations of hereditary hemorrhagic telangiectasia in the Afro-Caribbean population of the Netherlands Antilles: a family screening. Am J Med Genet. 2003;116A:324-8.

16. Bletz MJ. The First Dutch Settlement in Alberta: Letters from the Pioneer Years, 1903-1914 (review). Can Ethn Stud. 2007;39:3-6.

17. Donaldson J, McKeever T, Hall I, Hubbard R, Fogarty A. Complications and mortality in hereditary hemorrhagic telangiectasia: a population-based study. Neurology. 2015;84:1886-93.

18. Statistics Canada. 2009, Women and health. Statistics Canada Catalogue no. 89-503-X. Ottawa. Version updated May 2009. Ottawa. Available at: https://www150.statcan.gc.ca/n1/pub/89503-x/2010001/article/11543-eng.htm. Accessed June 13, 2018.

19 Shovlin CL, Chamali B, Santhirapala V, et al. Ischaemic strokes in patients with pulmonary arteriovenous malformations and hereditary hemorrhagic telangiectasia: associations with iron deficiency and platelets. PLoS One. 2014;9:e88812.

20. Shovlin C, Sodhi V, McCarthy A, Lasjaunias P, Jackson J, Sheppard M. Estimates of maternal risks of pregnancy for women with hereditary haemorrhagic telangiectasia (Osler-Weber-Rendu syndrome): suggested approach for obstetric services. BJOG. 2008;115:1108-15.

21. Velthius S, Buscarini E, van Gent MWF, et al. Grade of pulmonary right-to-left shunt on contrast echocardiography and cerebral complications: a striking association. Chest. 2013;144:542-8.

22. Mathis S, Dupuis-Girod S, Plauchu H, et al. Cerebral abscesses in hereditary haemorrhagic telangiectasia: a clinical and microbiological evaluation. Clin Neurol Neurosurg. 2012;114:235-40.

23. Liebeskind DS. Chapter 4: Intracranial collateral routes and anastomoses in interventional neuroradiology. Neurointerventional management: diagnosis and treatment. 2nd ed. Boca Raton: Taylor and Francis Group; 2012. p. 79.

24. Kristensen B, Malm J, Calberg B, et al. Epidemiology and etiology of ischemic stroke in young adults aged 18-44 years in Northern Sweden. Stroke. 1997;28:1702-9.

25. Maher CO, Piepgras DG, Brown RD, Friedman JA, Pollock BE. Cerebrovascular manifestations in 321 cases of hereditary hemorrhagic telangiectasia. Stroke. 2001;32:877-2.

26. Nataf F, Meder JF, Roux FX, et al. Angioarchitecture associated with haemorrhage in cerebral arteriovenous malformations: a prognostic statistical model. Neuroradiology. 1997;39:52-8.

27. International Classification of Diseases (ICD). World Health Organization. http://www.who.int/classificationsicd/en/. Accessed December 8, 2014. 